\title{
TRANSMISSION ELECTRON MICROSCOPY OF THE PRECLINICAL PHASE OF EXPERIMENTAL PHYTOPHOTODERMATITIS
}

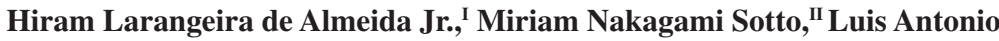 \\ Suita de Castro, ${ }^{\text {III }}$ Nara Moreira Rocha ${ }^{\text {III }}$
}

doi: $10.1590 / \mathrm{S1807-59322008000300014}$

Almeida Junior HL, Sotto MN, Castro LAS, Rocha NM. Transmission electron microscopy of the preclinical phase of experimental phytophotodermatitis. Clinics. 2008;63:371-4.

OBJECTIVE: To examine the epidermis in induced phytophotodermatitis using transmission electron microscopy in order to detect histologic changes even before lesions are visible by light microscopy.

INTRODUCTION: In the first six hours after the experimental induction of phytophotodermatitis, no changes are detectable by light microscopy. Only after 24 hours can keratinocyte necrosis and epidermal vacuolization be detected histologically, and blisters form by 48 hours.

METHODS: The dorsum of four adult rats (Rattus norvegicus) was manually epilated. After painting the right half of the rat with the peel juice of Tahiti lemon, they were exposed to sunlight for eight minutes under general anesthesia. The left side was used as the control and exposed to sunlight only. Biopsies were performed immediately after photoinduction and one and two hours later, and the tissue was analyzed by transmission electron microscopy.

RESULTS: No histological changes were seen on the control side. Immediately after induction, vacuolization in keratinocytes was observed. After one hour, desmosomal changes were also observed in addition to vacuolization. Keratin filaments were not attached to the desmosomal plaque. Free desmosomes and membrane ruptures were also seen. At two hours after induction, similar changes were found, and granular degeneration of keratin was also observed.

DISCUSSION: The interaction of sunlight and psoralens generates a photoproduct that damages keratinocyte proteins, leading to keratinocyte necrosis and blister formation.

CONCLUSIONS: Transmission electron microscopy can detect vacuolization, lesions of the membrane, and desmosomes in the first two hours after experimental induction of phytophotodermatitis.

KEYWORDS: Phytophotodermatitis. Animal Model. Transmission Electron Microscopy. Desmosomes. Keratinocytes.

\section{INTRODUCTION}

Phytophotodermatitis (PPD) is defined as a phototoxic reaction of the skin following contact with plant-derived substances (psoralens) and subsequent sunlight exposure. ${ }^{1-3}$

\footnotetext{
${ }^{\mathrm{I}}$ Department of Dermatology, Federal and Catholic University of Pelotas Pelotas/RS, Brazil.

II Department of Dermatology, University de São Paulo - São Paulo/SP, Brazil

III Laboratory of Electron Microscopy, EMBRAPA-CPA-CT, Pelotas/RS Brazil

hiramalmeidajr@hotmail.com

Received for publication on January 07, 2008

Accepted for publication on February 25, 2008
}

Clinically, the affected areas show erythema, blisters, or hyperpigmentation ${ }^{4-6}$. The disease occurs frequently in the summer months and its most common cause in South Brazil is the Tahiti lemon, where 16 cases per 100,000 inhabitants are estimated to occur each summer. ${ }^{7}$

PPD can be experimentally reproduced in rats using the peel juice of the Tahiti lemon. Very short sunlight exposure times of 2.5-10 minutes are sufficient to induce it. ${ }^{7}$ Clinical changes are visible after only 48 hours, and these involve erythema and desquamation. At 24 hours after induction, light microscopy reveals epidermal vacuolar degeneration and keratinocyte necrosis that increases with increasing exposure time. At 48 hours after induction, intra- 
and subepidermal blisters are visible. Nevertheless, light microscopy reveals no detectable changes within the first six hours after experimental induction.

The objective of this study was to determine whether transmission electron microscopy could be used to detect changes in the epidermis in induced PPD even before lesions were detectable by light microscopy.

\section{MATERIAL AND METHODS}

Hairs from the dorsum of four adult rats (Rattus norvegicus) were manually removed 72 hours before the experimental induction of PPD in order to avoid trauma artifacts. The rats were anaesthetized intramuscularly with $50 \mathrm{mg} / \mathrm{kg}$ of tiletamine hydrochloride and zolazepam hydrochloride. After painting the right half of each rat with undiluted peel juice from Tahiti lemon, they were exposed to summer sunlight (in February) for eight minutes at 11 a.m. in the city of Pelotas in the southern part of Brazil, at a latitude of $31.7^{\circ} \mathrm{S}$ and longitude of $52.3^{\circ} \mathrm{W}$.

The left side of each rat was used as the control and was exposed only to sunlight. Biopsies immediately after the experimental induction and after one and two hours were performed with 3-mm punches and processed for transmission electron microscopy using standard methods.

At 24 and 48 hours after induction, light microscopy was performed on the tissues to verify that the typical changes characteristic of PPD, including keratinocyte necrosis and blistering, were successfully reproduced.

This research was approved by the Ethics Committee of the Federal University of Pelotas.

\section{RESULTS}

No histological changes were seen on the control side of the rats at any of the three time points examined. The rat epithelial tissue was thinner than human epidermis. Desmosomes and hemidesmosomes with insertion of keratin filaments and the basal membrane could be easily identified.

Immediately after PPD induction, the skin showed keratinocyte cytoplasmatic vacuolization (Fig. 1a), and membrane ruptures were also visible near the sites of vacuolization. Some desmosomes contained keratin filaments that had lost contact with the desmosomal plaques (Fig. 1b).

One hour after the experimental procedure, the ultrastructural findings revealed more dramatic changes. Vacuolization was seen in the intercellular spaces, in the cytoplasm, and at the dermal-epidermal junction (Figs. 2a and $b$ ). In addition, desmosomal changes were also evident; in many areas keratin filaments were no longer attached to the desmosomal plaques and desmosomes were found freely in the sections (Figs. 3a and b). At higher magnifications, the desmosomal plaques were seen to be rounded, with the cell membrane folded over the plaque, and they were not binding desmosomes to one another (Figs. 4a and b). Membrane degeneration was also visible (Fig. 4b). Keratinocyte nuclei appeared normal.

At two hours after induction, vacuolization and free desmosomes were observed, similar to that at one hour after induction (Fig. 5a). Moreover the granular degeneration of keratin, which had lost its filamentous appearance, was visible by this point (Fig. 5b). In some areas the degenerated keratin was distributed parallel to the desmosomal plaques, which also showed signs of degeneration. Hemidesmosomes and their associated keratin filaments showed similar signs of degeneration, characterized by the granular aspect of the hemidesmosomal plaque and of the keratin. In contrast, nuclei still appeared normal at this point.

\section{DISCUSSION}

This experimental animal model can successfully reproduce PPD. In previous research using this model, light microscopy showed the epidermis to be normal during the first
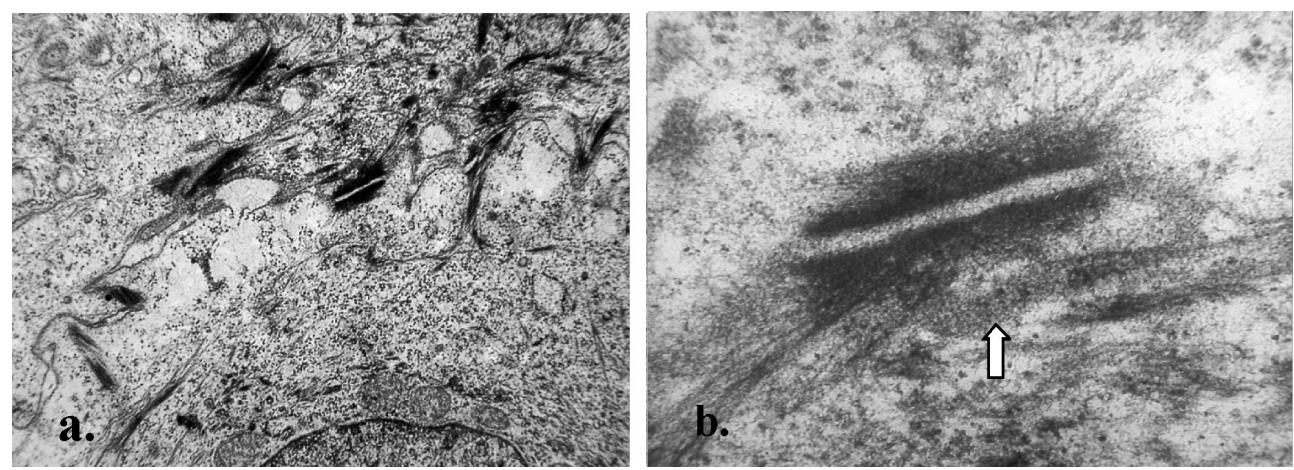

Figure 1 - Immediately after the experimental induction. a. Keratinocyte cytoplasmic vacuolization (x 28.000). b. Desmosome with loss of keratin anchorage in the inferior plaque (arrow) (x 40.000) 

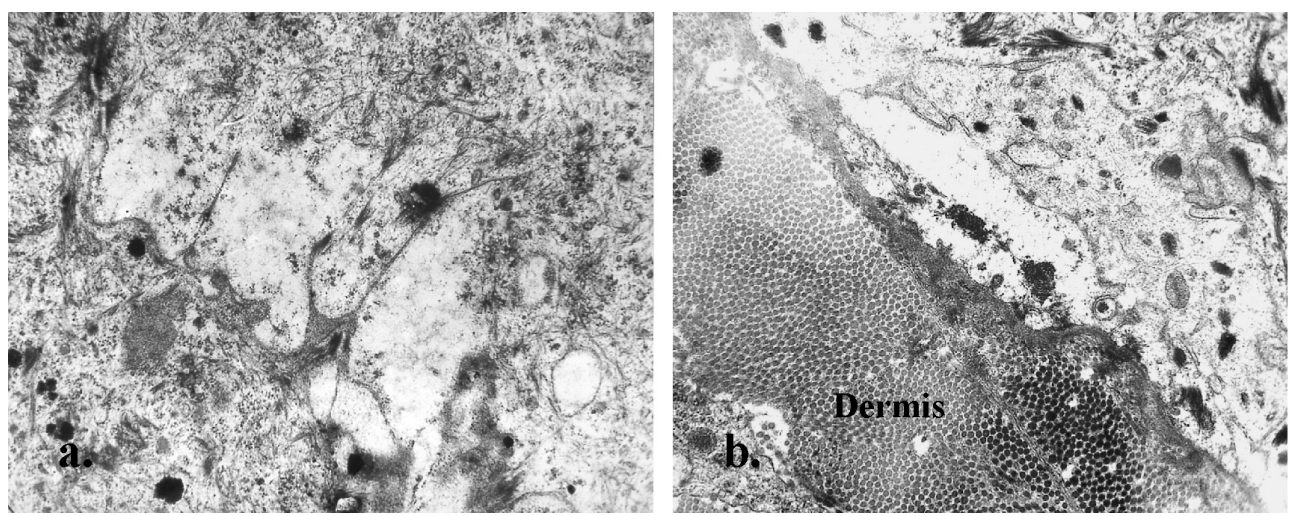

Figure 2 - One hour after the experimental induction a. Cytoplasmic and intercellular vacuolization (x 62.000). b. Vacuolization at the dermal-epidermal junction (x 62.000)
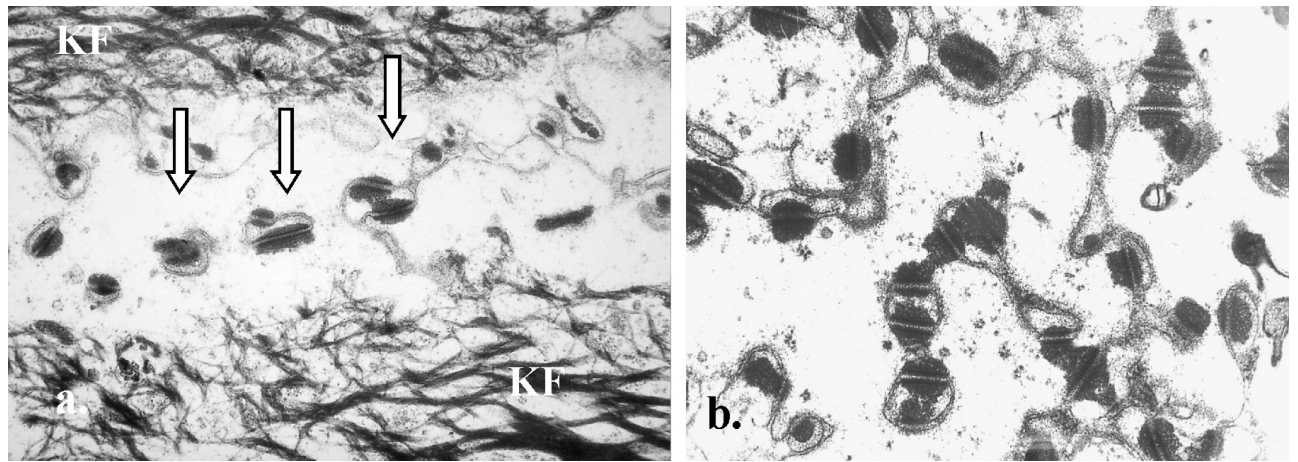

Figure 3 - One hour after the experiment a. Attachment loss of keratin filaments (KF) to the desmosomal plaques (arrows) (x 17.000). b. Free desmosomes without contact with keratin (x 60.000)
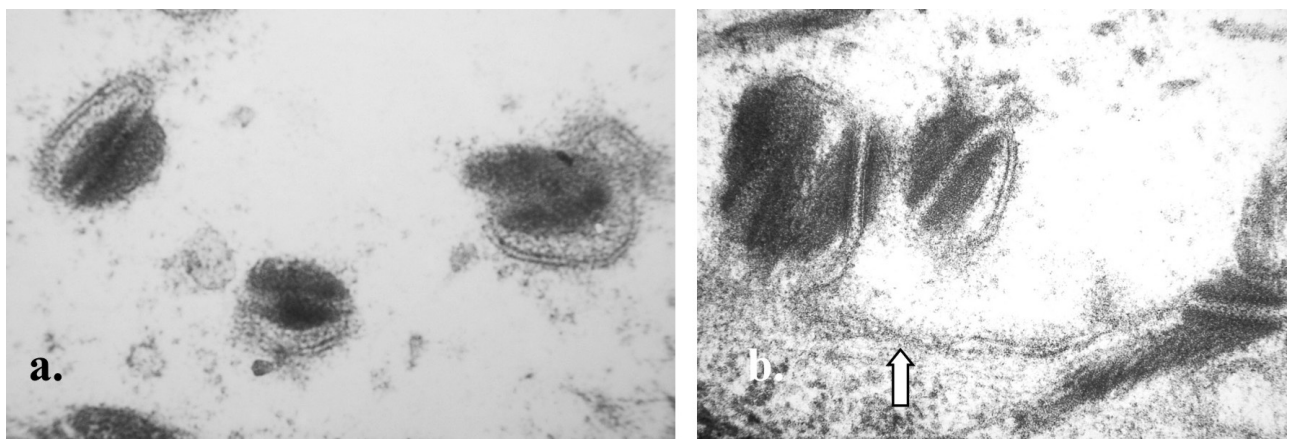

Figure 4 - One hour after induction a. Free desmosomes with curved cell membranes, which are not linking one desmosome to each other (x 120.000). b. Free desmosomes and membrane degeneration (arrow) (x 120.000)

6 hours after induction. ${ }^{7}$ The first signs of keratinocyte necrosis were detected at 24 hours, and they progressed to significant epidermal vacuolization and intra- and subepidermal blistering in 48 hours. The severity of the progression depended on the level of keratinocyte degeneration.

Erythema was observed after only 48 hours. These changes over time indicate that the interaction of sunlight and psoralens triggers cell death, which becomes detectable by light microscopy many hours later, and takes even longer to manifest clinically. This delay is in agreement with clinical experience, where patients report an interval between handling vegetables outside and the onset of lesions.

The aim of this study was to examine epidermal changes in PPD using the more sensitive method of transmission electron microscopy. We hypothesized that this technique would detect PPD symptoms in the first hours after induction, when light microscopy still shows normal skin.

Using TEM, cytoplasmatic vacuolization was detected immediately after skin exposure to sunlight in the presence of Tahiti lemon peel juice, which became more intense after one hour. At the same time, desmosomal changes were also observed, and they involved the loss of keratin anchorage in 

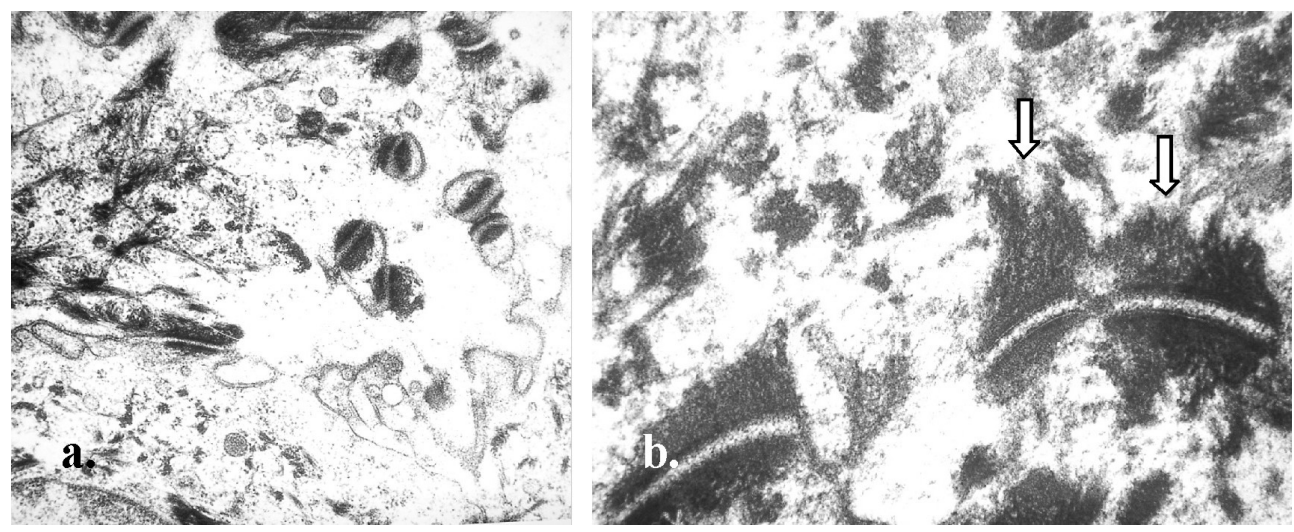

Figure 5 - Two hours after the experiment a. Intercelular vacuolization with free desmosomes (x 25.000). b. granular degeneration of keratin filaments (arrows), which lost its typical filamentous aspect (x 78.000)

the desmosomal plaque, leading to free desmosomes in the examined sections. Membrane degeneration was also seen.

Two hours after PPD induction, granular degeneration of the keratin filaments and of the desmosomal plaques were observed in addition to the previously detected free desmosomes and vacuolization.

Probably the cell membranes, the proteins involved in the adhesion of keratin filaments in the desmosomal plaque and keratin are more sensitive to the photoproduct made when sunlight and psoralens interact, since only these were the only subcellular structures showing changes during the preclinical phase after induction. These protein lesions lead to keratinocyte death within 24 hours of induction, and to blisters within 48 hours. These informations do not support a previous concept, that nuclear changes could be the cause of cell necrosis in phototoxic reactions. ${ }^{3}$

The desmosomal plaque is formed by a complex network of proteins ${ }^{8}$ involved not only in adhesion of keratinocytes to one another, but also in cytoskeletal organization, ${ }^{9}$ which may explain the signs of necrosis and not acantholytic changes.

Very little information is available on the ultrastructural aspects of desmosome lesions, which can have genetic ${ }^{10,11}$ or autoimmune ${ }^{12}$ origins. One report on a case of desmoplakin mutation, a protein found in the desmosomal plaque, showed desmosomes without anchoring keratin filaments ${ }^{10}$, similar to the free desmosomes found in this research, which suggests that desmoplakin may also be damaged in PPD.

\section{REFERENCES}

1. Stoner JG, Rasmussen JE. Plant dermatitis. J Am Acad Dermatol. 1983;9:1-15.

2. Diógenes MJN, Matos FJA. Dermatite de Contato por Plantas. An Bras Dermatol. 1999;74:629-34.

3. Tunget CL, Turchen SG, Monoguerra AS, Clark RF, Pudoff DE. Sunlight and the plant: a toxic combination: severe phytophotodermatitis from Cneorium dumosun. Cutis. 1993;54:400-2.

4. Ahamed I, Holmes RC. Phytophotodermatitis mimicking superficial lymphangitis. Br J Dermatol. 2000;142:1047-70.

5. Solis RR, Dotson DA, Trizna Z. Phytophotodermatitis. A sometimes difficult diagnosis. Arch Fam Méd. 2000;9:1195-6.

6. Carlsen K, Weismann K. Phytophotodermatitis in 19 children admitted to hospital and their differential diagnoses: Child abuse and herpes simplex virus infection. J Am Acad Dermatol. 2007;57:S88-91.

7. Gonçalves NEL, de Almeida Jr. HL, Hallal EC, Amado M. Experimental phytophotodermatitis. Photodermatol Photoimmunol Photomed. $2005 ; 21: 318-21$
8. McGrath JA, Wessagowit V. Human hair abnormalities resulting from inherited desmosome gene mutations. Keio J Med. 2005;54:72-9.

9. Lechler T, Fuchs E. Desmoplakin: an unexpected regulator of microtubule organization in the epidermis. J Cell Biol. 2007;176:147-54.

10. Jonkman MF, Pasmooij AM, Pasmans SG, van den Berg MP, Ter Horst HJ, Timmer A, et al. Loss of desmoplakin tail causes lethal acantholytic epidermolysis bullosa. Am J Hum Genet. 2005;77:653-60. Epub 2005 Aug 17.

11. McMillan JR, Haftek M, Akiyama M, South AP, Perrot H, McGrath JA, et al. Alterations in desmosome size and number coincide with the loss of keratinocyte cohesion in skin with homozygous and heterozygous defects in the desmosomal protein plakophilin 1. J Invest Dermatol. 2003;121:96-103.

12. Shimizu A, Ishiko A, Ota T, Tsunoda K, Amagai M, Nishikawa T. $\mathrm{IgG}$ binds to desmoglein 3 in desmosomes and causes a desmosomal split without keratin retraction in a pemphigus mouse model. J Invest Dermatol. 2004;122:1145-53. 